\title{
Fuzzy expert system in the prediction of neonatal resuscitation
}

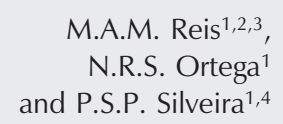

M.A.M. Reis ${ }^{1,2,3}$,

N.R.S. Ortega ${ }^{1}$ and P.S.P. Silveira ${ }^{1,4}$

\author{
${ }^{1}$ Informática Médica, Faculdade de Medicina, Universidade de São Paulo, \\ São Paulo, SP, Brasil \\ ${ }^{2}$ Informática Médica, Departamento de Medicina, Universidade Regional de Joinville, \\ Joinville, SC, Brasil \\ ${ }^{3}$ Departamento de Neonatologia, Maternidade Darcy Vargas, Joinville, SC, Brasil \\ ${ }^{4}$ Decision System Group, Harvard/MIT, Health, Science and Technology, \\ Cambridge, MA, USA
}

\section{Correspondence \\ N.R.S. Ortega \\ Informática Médica \\ Faculdade de Medicina, USP \\ 05508-900 São Paulo, SP \\ Brasil}

Presented at the 1st Symposium on Advances in Medical Research, Institute of Medical Investigation Laboratories, HC-FMUSP, São Paulo, SP, Brazil, March 21-22, 2003.

Received June 12, 2003

Accepted February 3, 2004
..........................

\begin{abstract}
In view of the importance of anticipating the occurrence of critical situations in medicine, we propose the use of a fuzzy expert system to predict the need for advanced neonatal resuscitation efforts in the delivery room. This system relates the maternal medical, obstetric and neonatal characteristics to the clinical conditions of the newborn, providing a risk measurement of need of advanced neonatal resuscitation measures. It is structured as a fuzzy composition developed on the basis of the subjective perception of danger of nine neonatologists facing 61 antenatal and intrapartum clinical situations which provide a degree of association with the risk of occurrence of perinatal asphyxia. The resulting relational matrix describes the association between clinical factors and risk of perinatal asphyxia. Analyzing the inputs of the presence or absence of all 61 clinical factors, the system returns the rate of risk of perinatal asphyxia as output. A prospectively collected series of 304 cases of perinatal care was analyzed to ascertain system performance. The fuzzy expert system presented a sensitivity of $76.5 \%$ and specificity of $94.8 \%$ in the identification of the need for advanced neonatal resuscitation measures, considering a cut-off value of 5 on a scale ranging from 0 to 10 . The area under the receiver operating characteristic curve was 0.93 . The identification of risk situations plays an important role in the planning of health care. These preliminary results encourage us to develop further studies and to refine this model, which is intended to implement an auxiliary system able to help health care staff to make decisions in perinatal care.
\end{abstract}

Key words

- Fuzzy sets

- Risk analysis

- Medical expert systems

- Perinatal asphyxia

- Neonatal resuscitation

......................

\section{Introduction}

The World Health Organization estimates that 3.6 million infants born every year in developing countries develop birth asphyxia, requiring resuscitation. Approximately 900,000 of these newborns die annually and an unknown number remain with long-term neurologic sequelae as a result of such phenomenon occurring before, during or immediately after birth (1). These events, more frequently caused by improper intrauterine transfer of maternal blood oxygen to the fetus resulting in an acute inadequate supply of oxygen to the fetal organs and tissues, are not always predictable or preventable and 
frequently lead to the birth of a severely depressed baby who needs immediate skilled resuscitation in the delivery room. For this reason, it is common sense in neonatal resuscitation practice, and a strict recommendation of the Neonatal Resuscitation Program, that any birth should be assisted by a specially trained and skilled professional to correctly perform all the maneuvers necessary to rescue a severely asphyxiated newborn.

However, for operational and economical reasons, it is rarely possible to follow this recommendation in low demand hospitals and in developing countries $(2,3)$ where the prediction of delivery of an asphyxiated infant would be critically important to help plan and optimize patient care by the best skilled personnel available. Even in high complexity teaching hospitals in developed countries, which usually follow this recommendation, the anticipation of a high risk situation could automatically alert the best trained neonatal resuscitation team.

Researchers have been working on identifying risk factors and creating ways of predicting poor outcomes in perinatal medicine for a long time (4-7). Some important initiatives were directed at assessment of risk for perinatal mortality (8) and very low birth weight (9). Efforts have been made to identify the severity of neonatal illness and to create predictor systems of morbidity and mortality known as "neonatal risk scoring systems", such as the score for neonatal acute physiology and the clinical risk for babies score $(10,11)$, especially useful for severity of illness correction in the comparison of results obtained at different neonatal units. However, no published data are available about predictor systems for perinatal asphyxia or neonatal resuscitation needs.

In fact, as observed in other areas, decision support is a fecund substratum for the creation of systematic techniques in medicine. Impressive advances have been noticed in the development of the so-called expert or cognitive systems suggested for diagnostic and decision making analysis, mimicking the mental procedures of human experts in specific areas of knowledge as a way to simulate the memory and judgment of specialists. The evident importance of such expert systems would be to make the specialists' knowledge and experience available any time in places where they are critically needed but usually unavailable.

On the way to guarantee more precise and safer health care procedures, decision support systems are increasingly present in critical sets such as intensive care units and emergency departments (12).

The logical analysis of the diagnostic process makes medical diagnostic decision amenable to being mimicked by computer systems. The development of such systems is part of the investigation field called artificial intelligence, which can be defined as "the branch of computer science that is concerned with the automation of intelligent behaviour" (13). Over the last decades, the increasing capacity of computers to process and store data and to carry out complex logical manipulations, in association with networks able to promptly make data and results available at a distance, has encouraged the dissemination of medical knowledge (14). Mathematical and statistical techniques including decision trees, discriminate analysis and cluster analysis, among others, have been already applied in many classic expert systems. More recently, the fuzzy sets theory has been successfully applied in medicine, particularly in the development of fuzzy expert systems (15-20).

For the creation of decision support systems, great emphasis is placed on the interpretation of the natural language ordinarily used in clinical practice. The use of common but subjective terms like "worse", "better", "stable", "a little", "a lot", "moderate", "discretely" and "intense", and even the no less subjective cross code used in descriptions of signs and symptoms exemplify the vagueness of data to be manipulated. To qualita- 
tively and quantitatively deal with such extreme inexactness, specific tools should be available $(12,19)$. The fuzzy sets theory represents one of the most prominent progresses in this area.

Since developing and applying computers as auxiliary diagnostic tools depend on a multidisciplinary approach, a brief review of some basic concepts of fuzzy theory is necessary for the sake of best understanding, as described below.

\section{Fuzzy sets theory}

The theory of fuzzy sets was introduced by Lotfi A. Zadeh, University of California, Berkeley, in the 1960's as a means to model the uncertainty within natural language. The mechanics of fuzzy sets theory was set forth in 1965 (21), based on Zadeh's key notion of graded membership, according to which a set could have members that belong to it only in part. Such fuzzy sets have imprecise boundaries and therefore gradual transition from membership to non-membership of an element in its fuzzy set is observed. The ambition of fuzzy sets is to provide interaction of natural language and numerical models (22).

Thus, if we assume that $X$ is a set serving as the universe of discourse, a fuzzy subset $A$ of $X$ is associated with a characteristic function:

$\mu_{\mathrm{A}}: X \rightarrow[0,1]$

Eq. 1

which is generally called membership function. Then, for each $x, \mu_{\mathrm{A}}(x)$ indicates to what extent $x$ is a member of set $A$. This membership degree indicates the compatibility degree of the assertion " $x$ is $A$ ". Using a clinical example: considering that someone has a fever when having a body temperature above $37.0^{\circ} \mathrm{C}$, it is easy to understand that a patient with $38^{\circ} \mathrm{C}$ has a fever (membership degree of 1.0). But we could say, based on the fuzzy sets theory, that two patients with temperatures of $36.8^{\circ} \mathrm{C}$ and $37.0^{\circ} \mathrm{C}$ are members of the "febrile patient set" too, with a membership degree of 0.8 and 0.9 , respectively.

The classic set theoretical operations can be extended to fuzzy sets, which have membership grades in the interval (0.1). So, if we assume that $A$ and $B$ are two fuzzy subsets of $X$, their union is a fuzzy subset $C$ of $X$, denoted $C=A \cup B$ identified by the maximum (max) value between $A(x)$ and $B(x)$, such that for each $x$ in $X(22)$ :

$C(\mathrm{x})=\max [A(x), B(x)] \quad$ Eq. 2

In addition, their intersection is another fuzzy subset $D$ of $X$, denoted $D=A \cap B$ identified by the minimum (min) value between $A(x)$ and $B(x)$, such that for each $x$ in $X$ (22):

$D(x)=\min [A(x), B(x)] \quad$ Eq. 3

Another important concept in fuzzy sets is fuzzy relations. A fuzzy relation $\mathrm{R}$ between two crisp sets $X=\{x\}$ and $Y=\{y\}$ is defined as a fuzzy set in the Cartesian product $X \times Y$, i.e.,

$R=\left\{\mu_{R}(x, y) /(x, y)\right\} \quad$ Eq. 4

for each $(x, y) \in X \times X$, where $\mu_{R}(x, y): X \times 1 \rightarrow$ $[0,1]$ is the membership function of the fuzzy relation $\mathrm{R}$, and $\mu_{R}(x, y) \in[0,1]$ gives the degree to which the elements $x \in X$ and $y \in$ $Y$ are in relation $\mathrm{R}$ to each other. Since this basic type of a fuzzy relation is defined in the Cartesian product of two sets, such a fuzzy relation is sometimes called a binary fuzzy relation. However, this concept could be generalized to $n$ dimensions of fuzzy relations $(21,22)$. A fuzzy relation could express a partial (imprecise) relationship between elements of some sets, as opposed to a precise one in the case of a crisp relation in which any elements can either be related or not. In the fuzzy relation we are concerned about gradual relationships, from 1 for being fully in relation to 0 for not being in relation at all, through all intermediate values. Fuzzy relations could be represented in a matrix form, which usually simplifies the composi- 
tion of fuzzy relation methods.

One of the most useful composition of fuzzy relations is the so-called max-min composition. The max-min composition of two fuzzy relations $\mathrm{R}$ in $X \times Y$ and $\mathrm{S}$ in $Y \times Z$, written $R \mathrm{o}_{\text {max-min }} S$ is defined as a fuzzy relation in $X \times \mathrm{x} Z$ such that

$$
\mu_{\text {Romax-minS }}(x, y)=\max _{y \in \mathrm{Y}}\left[\min \left(\mu_{R}(x, y), \mu_{S}(y, z)\right)\right]
$$

for each $x \in X$ and $\mathrm{z} \in Z$. The mathematical operation expressed above is similar to a multiplication of matrices, where each matrix represents a fuzzy relation $(22,23)$. The use of the above equation will be clarified with an example in the Material and Methods section.

The objective of the present study was to demonstrate the viability of using fuzzy sets theory to correlate the natural language used in perinatology with a numerical model which can be used in a decision support system to simulate the risk analysis made by expert judgment.

\section{Material and Methods}

\section{Definition of risk factors}

A list of antepartum and intrapartum conditions associated with overall risk to new-

\begin{tabular}{|c|c|c|}
\hline Score & Risk & Observation \\
\hline 0 & - & $\begin{array}{l}\text { The risk of needing ANRM is the same as that of the } \\
\text { unexposed population }\end{array}$ \\
\hline 1 & Extremely low & $\begin{array}{l}\text { The possibility of needing ANRM is remote, but slightly } \\
\text { higher than observed in the unexposed population }\end{array}$ \\
\hline 2 & Very low & - \\
\hline 3 & Low & The need of ANRM is less than expected \\
\hline 4 & Medium-low & - \\
\hline 5 & Medium & Will probably need ANRM \\
\hline 6 & Medium-high & - \\
\hline 7 & High & The need for ANRM is strongly expected \\
\hline 8 & Very high & - \\
\hline 9 & Extremely high & The possibility of not needing ANRM is remote \\
\hline 10 & - & Will surely need ANRM \\
\hline
\end{tabular}

Table 1. Reference risk scoring system.

Scores without an observation indicate the classification between two categories ANRM $=$ advanced neonatal resuscitation measures. borns presented in the medical literature (24) was reviewed and detailed by the neonatologist researcher. The focus of this review was the identification of factors more strongly related to the occurrence of perinatal asphyxia.

After the exclusion of four conditions (neurologic maternal illness, drug therapy, no prenatal care, and precipitous labor) and maintenance, for calibration purposes, of three low risk conditions (hypothyroidism, hyperthyroidism and anemia), a list of the 61 remaining conditions was submitted on paper to nine neonatologists specially trained and skilled in neonatal resuscitation according to international standards. These neonatologists are part of a neonatology team regularly working in delivery room care with a previous experience of directly assisting more than 60,000 newborns over the last ten years.

The experts were asked to analyze each of the 61 factors separately and to quantify (with scores from 0 to 10) the expected risk of needing advanced neonatal resuscitation in the delivery room for the newborn of a hypothetically exposed pregnant woman. The given score was assumed as a degree of association between the presence of the factor and the risk of perinatal asphyxia, i.e., in the model context, this score represents the intensity of the fuzzy relation between clinical finding and perinatal asphyxia occurrence. They were told to answer each topic as if that information was the only one available during their ward activity. As a reference guide to risk analysis, the neonatologists were asked to consider the scoring system shown in Table 1.

Tables 2 and 3 show the process of review and selection of risk factors analyzed by the experts. In the third column the degree of association explained above is presented, which was considered a fuzzy degree in the matrix of fuzzy relations.

For study purposes, we considered as need of advanced neonatal resuscitation measures (ANRM) any condition in which 
positive pressure ventilation and/or endotracheal intubation and/or chest compression and/or medications were necessary during immediate postpartum neonatal care, requiring the presence of a specially skilled professional. In this study, we did not include clinical situations which would need basic resuscitation efforts such as tactile stimulation and oxygen inhalation, that could be executed by a less skilled professional.

Since the need of resuscitation efforts for a depressed delivered newborn has a close etiological relation to perinatal asphyxia, and its importance to the perinatal care planning, we chose the need for advanced neonatal resuscitation maneuvers as the study marker of perinatal asphyxia. Since a score of 5 was related to medium risk according to the proposed Reference Risk Score System (Table 1), this score was defined as the theoretical cut-off value for the "highest risk of ANRM occurrence".

\section{Expert fuzzy system}

The median of score assigned to each factor was firstly divided by 10 , transforming the possible range of values from 1 to 10 , to 0.1 to 1.0 (Tables 2 and 3). The median of the given scores was used to minimize the effect of

Table 2. Analysis of maternal early gestational risk situations.

\begin{tabular}{|c|c|c|}
\hline Literature risk conditions & Reviewed risk factors & Relation matrix \\
\hline \multirow[t]{3}{*}{ Maternal diabetes } & Maternal diabetes, type 1 & 0.2 \\
\hline & Maternal diabetes, type 2 & 0.1 \\
\hline & Maternal diabetes, gestational & 0.1 \\
\hline \multirow[t]{3}{*}{ Pregnancy-induced hypertension } & Pregnancy-induced hypertension, mild & 0.1 \\
\hline & Pregnancy-induced hypertension, severe & 0.6 \\
\hline & Eclampsia & 0.7 \\
\hline \multirow[t]{2}{*}{ Chronic hypertension } & Chronic hypertension, mild & 0.2 \\
\hline & Chronic hypertension, severe & 0.4 \\
\hline \multirow[t]{2}{*}{ Chronic maternal illness, cardiovascular } & Maternal cardiovascular illness, compensated & 0.2 \\
\hline & Maternal cardiovascular illness, uncompensated & 0.6 \\
\hline \multirow[t]{2}{*}{ Chronic maternal illness, thyroid } & Maternal hyperthyroidism & 0.1 \\
\hline & Maternal hypothyroidism & 0.1 \\
\hline \multirow[t]{3}{*}{ Chronic maternal illness, pulmonary } & Maternal pulmonary obstructive disease & 0.2 \\
\hline & Maternal asthma, compensated & 0.1 \\
\hline & Maternal asthma, crisis & 0.4 \\
\hline Chronic maternal illness, renal & Maternal chronic renal insufficiency & 0.3 \\
\hline \multirow[t]{3}{*}{ Maternal illness, anemia or isoimmunization } & Anemia & 0.1 \\
\hline & Isoimmunization without hydrops fetalis & 0.3 \\
\hline & Isoimmunization with hydrops fetalis & 0.7 \\
\hline \multirow[t]{2}{*}{ Previous fetal or neonatal death } & Previous fetal death & 0.2 \\
\hline & Previous neonatal death & 0.2 \\
\hline \multirow[t]{2}{*}{ Maternal infection } & Maternal urinary infection, without treatment & 0.2 \\
\hline & Maternal urinary infection, during treatment & 0.1 \\
\hline \multirow[t]{2}{*}{ Bleeding in second or third trimester } & Previous uterine bleeding & 0.3 \\
\hline & Active uterine bleeding, without etiologic diagnosis & 0.5 \\
\hline Polyhydramnios & Polyhydramnios & 0.3 \\
\hline Oligohydramnios & Oligohydramnios & 0.3 \\
\hline Multiple gestation & Multiple gestation & 0.5 \\
\hline \multirow[t]{2}{*}{ Age $<16$ or $>35$ years } & Age $<16$ & 0.2 \\
\hline & Age $>35$ & 0.2 \\
\hline Maternal substance abuse & Maternal substance abuse & 0.3 \\
\hline
\end{tabular}

The process of selection and evaluation of clinical conditions by neonatologists from the reference list obtained in the literature to the revised list presented here to the specialists, and median scores assigned to each condition. 
eventual discrepancies among the experts' opinions and represented the grade of risk perceived by this group. As discussed above, these values represented the degree of association between clinical signs and risk of perinatal asphyxia. The aggregation of all these values forms the fuzzy relational matrix, a matrix with 61 lines and one column.

The fuzzy relational findings/risk matrix represents the knowledge of experts in the model. It is used by the system to compute the perinatal asphyxia risk for any case analyzed by the use of a state matrix built for each case. The state matrix that identifies the presence (value $=1$ ) or absence (value $=0$ ) of each of all the risk factors observed by the user is the input of the model. Note that the system input is a crisp rather than a fuzzy value in order to simplify the actual utilization of this system in a perinatal unit. Then, for each state matrix, the system computes the risk value through the fuzzy max-min composition described in the fuzzy sets theory section.

The system output is found through the composition between clinical findings and the respective risk matrix. The mathematical process is similar to a matrix multiplication when, in this case, the sum is changed by the minimum operator and the multiplication is changed by the maximum operator. The result is a unitary matrix (one line and one column) whose value, normalized to 0 to 10 ,

Table 3. Analysis of fetal and perinatal risk situations.

\begin{tabular}{|c|c|c|}
\hline Literature risk conditions & Reviewed risk factors & Relation matrix \\
\hline \multirow{2}{*}{ Fetal malformation } & Fetal malformation, cardiovascular/pulmonary/CNS & 0.6 \\
\hline & Fetal malformation, others & 0.3 \\
\hline Diminished fetal activity & Diminished fetal activity & 0.5 \\
\hline \multirow[t]{2}{*}{ Size-dates discrepancy } & Intrauterine growth restriction & 0.4 \\
\hline & Fetal macrosomy & 0.3 \\
\hline Post-term gestation & Post-term gestation & 0.3 \\
\hline Emergency cesarean section & Emergency cesarean section & 0.7 \\
\hline Forceps or vacuum-assisted delivery & Forceps or Kristeller maneuver-assisted delivery & 0.4 \\
\hline \multirow[t]{2}{*}{ Breech or other abnormal presentation } & Breech presentation, cesarean section delivery & 0.4 \\
\hline & Breech presentation, vaginal delivery & 0.6 \\
\hline \multirow[t]{3}{*}{ Premature labor } & Premature labor $<30$ weeks & 0.9 \\
\hline & Premature labor $30-33$ weeks & 0.6 \\
\hline & Premature labor $34-36$ weeks & 0.4 \\
\hline \multirow[t]{2}{*}{ Chorioamnionitis } & Maternal fever, intrapartum & 0.3 \\
\hline & Foul smelling amniotic fluid & 0.3 \\
\hline \multirow[t]{2}{*}{ Prolonged rupture of membranes ( $>18 \mathrm{~h}$ before delivery) } & Prolonged rupture of membranes ( $24-48 \mathrm{~h}$ before delivery) & 0.4 \\
\hline & Prolonged rupture of membranes ( $>48 \mathrm{~h}$ before delivery) & 0.5 \\
\hline Prolonged labor (>24 h) & Prolonged labor (>24 h) & 0.5 \\
\hline Prolonged second stage of labor ( $>2 \mathrm{~h}$ ) & Prolonged second stage of labor ( $>2 \mathrm{~h}$ ) & 0.6 \\
\hline Fetal bradycardia & Fetal bradycardia & 0.7 \\
\hline \multirow[t]{2}{*}{ Non-reassuring fetal heart rate patterns } & Fetal tachycardia & 0.5 \\
\hline & Undetectable fetal heart activity & 1.0 \\
\hline Use of general anesthesia & Use of general anesthesia & 0.7 \\
\hline Uterine tetany & Uterine tetany & 0.7 \\
\hline Narcotics administered to mother within $4 \mathrm{~h}$ of delivery & Narcotics administered to mother within $4 \mathrm{~h}$ of delivery & 0.8 \\
\hline \multirow[t]{2}{*}{ Meconium-stained amniotic fluid } & Meconium-stained amniotic fluid, thin & 0.2 \\
\hline & Meconium-stained amniotic fluid, thick & 0.8 \\
\hline Prolapsed cord & Prolapsed cord & 0.9 \\
\hline Abruptio placentae & Abruptio placentae & 0.9 \\
\hline Placenta previa & Placenta previa & 0.6 \\
\hline
\end{tabular}

The process of selection and evaluation of clinical conditions by neonatologists from the reference list obtained in the literature to the revised list presented here to the specialists, and median scores assigned to each condition. CNS $=$ central nervous system. 
represents a score of the risk of needing ANRM associated with the case. Figure 1 shows an example of use of maximum-minimum composition during a hypothetical case analysis by the system. Observe that the first step is the selection of the minimum values between the paired input and the respective degree of association in the finding/risk matrix. The composition is completed with the selection of the maximum value of all the resulting (minimum) values. The range of the example presented does not show all the possible risk factors, but only those related to the case.

It is important to note how simple and fast the calculation of fuzzy relation composition is. This makes it feasible to develop expert system software and to apply it to the real perinatal unity environment.

\section{Experimental analysis}

As a way of evaluating the performance of the developed fuzzy expert system in risk analysis, a prospective follow-up of a series of cases requiring perinatal care was done at a reference tertiary perinatal medical center in southern Brazil from October to December 2002. The protocol was approved by the hospital Ethics Committee and written informed consent was obtained from the persons responsible for the subjects.

Each pregnant woman admitted to the hospital during the study period was invited to participate. Refusal to have her information included in the study, occurrence of abortion or stillbirths, and not giving birth during the study period were the exclusion criteria.

Data were collected by the same researcher from interviews of patients, health professionals and from patient medical records. Information was recorded from the time of patient admission to the first assistance of the newborn after birth, including aspects of previous maternal diseases and clinical conditions occurring during gestation, labor and delivery. Special emphasis was placed on medical interventions and mother and newborn outcomes. Twins were analyzed separately, so the occurrence of ANRM in either child was considered.

The collected cases were then analyzed by the developed fuzzy expert system to compare the estimated risks with the outcome observed and the sensitivity and specificity of the model were determined based on the data analyzed. The operating characteristics were plotted and graphically analyzed on the receiver operating characteristic (ROC) curve, which informs us about the estimated performance of the fuzzy expert model in predicting the need for ANRM in the data sample considered.

\section{Results}

The nine neonatologists had no signifi-

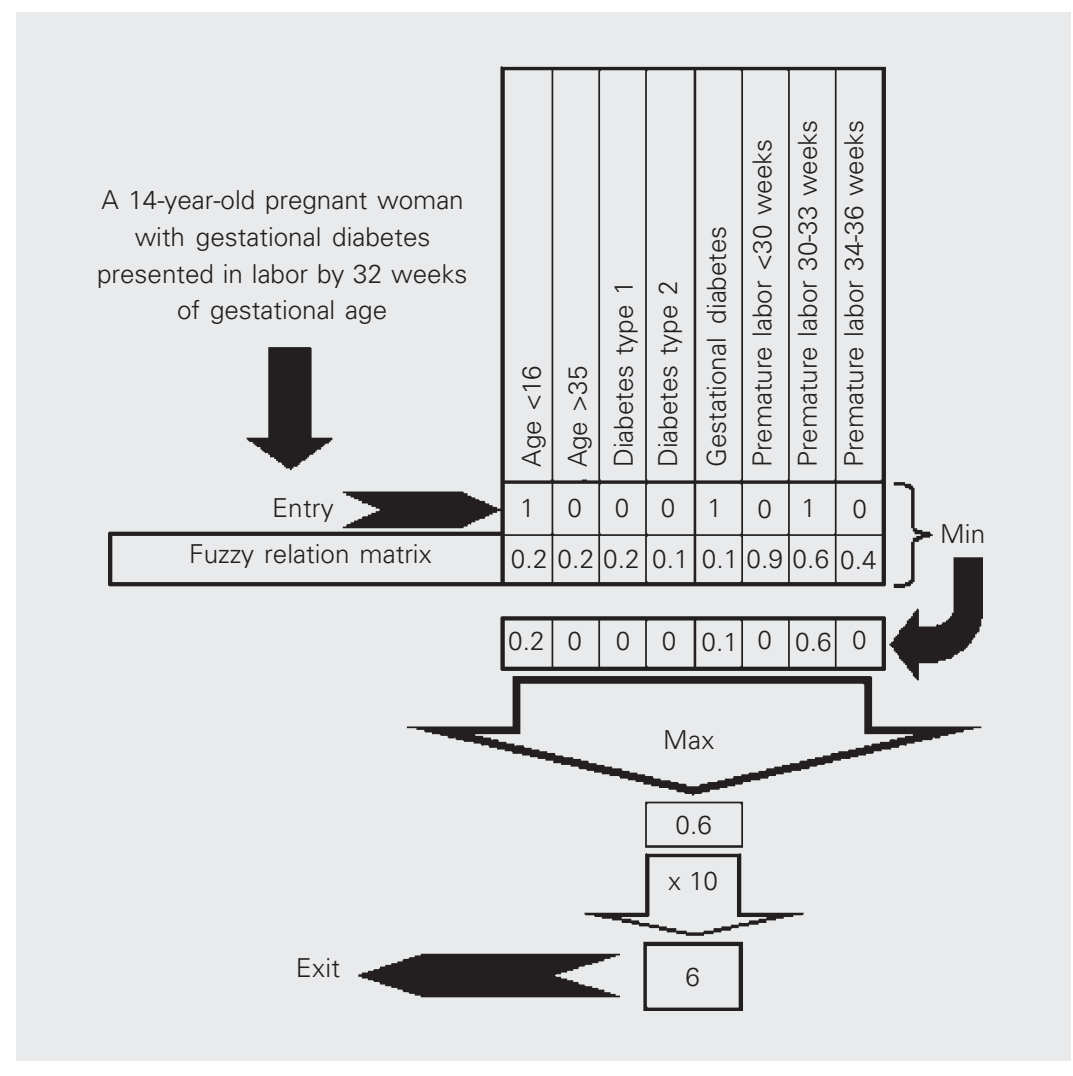

Figure 1. Example of fuzzy maximum-minimum (max-min) composition performance. A hypothetical clinical example shows the rationale of the model, using a small part of the knowledge bank for demonstration purposes. 
Table 4. Characteristics of the study sample.

\begin{tabular}{|c|c|c|}
\hline Maternal characteristics ( $N=303$ ) & N & $\%$ \\
\hline \multicolumn{3}{|l|}{ Medical } \\
\hline Anemia & 63 & (20.8) \\
\hline Previous spontaneous abortion & 52 & (17.1) \\
\hline Preeclampsia & 17 & (5.6) \\
\hline Age of above 35 & 17 & (5.6) \\
\hline Gestational diabetes & 13 & (4.2) \\
\hline Chronic hypertension & 11 & (3.6) \\
\hline \multicolumn{3}{|l|}{ Obstetric } \\
\hline Parity 0 & 102 & (33.7) \\
\hline Parity $\geq 1$ & 201 & (66.3) \\
\hline \multicolumn{3}{|l|}{ Delivery } \\
\hline Vaginal & 205 & $(67.7)$ \\
\hline Cesarean section & 99 & (32.7) \\
\hline Epidural analgesia & 55 & $(18.2)$ \\
\hline Forceps & 10 & (3.3) \\
\hline Kristeller maneuver & 50 & (16.5) \\
\hline Neonatal characteristics $(N=304)$ & N & $\%$ \\
\hline \multicolumn{3}{|l|}{ Outcome } \\
\hline 1-min Apgar $<7$ & 24 & (7.9) \\
\hline 5-min Apgar $<7$ & 7 & (2.3) \\
\hline Death & 1 & (0.3) \\
\hline Intensive/Intermediate care unit & 19 & (6.3) \\
\hline Rooming-in care & 284 & (93.4) \\
\hline \multicolumn{3}{|l|}{ Resuscitation measures } \\
\hline Positive pressure ventilation & 15 & (4.9) \\
\hline Endotracheal intubation & 5 & (1.6) \\
\hline Chest compressions & 1 & (0.3) \\
\hline Resuscitation medications & 2 & (0.7) \\
\hline
\end{tabular}

cant difficulties in scoring the factors, but all of them occasionally used the reference risk scoring system as a helping tool during risk analysis.

Information was collected about 303 pregnant women who gave birth to 304 newborns (one twin gestation) during the study period. Mean maternal age was $25.18 \pm 6.1$ years $(13$ to 43$)$. Most of the women were multiparous $(66.3 \%)$ and $18.1 \%$ of them received epidural analgesia during labor. The cesarean rate was $32.6 \%$.

Table 4 summarizes the most important characteristics of the sample studied.

Only one mother needed intensive care after delivery as a consequence of her previous disease. Of the 304 newborns, 273 were term and $31(10.2 \%)$ were preterm babies (eight below 34 weeks of gestational age). Mean birth weight was 3,233.5 $\pm 614.7 \mathrm{~g}$ (525 to $5,045 \mathrm{~g})$. Ninety-one (29.9\%) infants received oxygen inhalation and 17 (5.6\%) received ANRM immediately after birth. Only $7(2.3 \%)$ newborns had a fifth minute Apgar score below 7. One extremely premature infant died in the delivery room and 19 newborns $(6.3 \%)$ needed special care (intermediate or intensive care) after first assistance in the delivery room.

The formerly proposed cut-off value of 5

\begin{tabular}{ccc} 
Cut-off value & Sensitivity & Specificity \\
\hline 0.0 & 100.0 & 27.9 \\
0.1 & 100.0 & 41.8 \\
0.2 & 100.0 & 59.6 \\
0.3 & 94.1 & 72.5 \\
0.4 & 76.5 & 92.0 \\
0.5 & 76.5 & 94.8 \\
0.6 & 47.1 & 96.9 \\
0.7 & 29.4 & 98.3 \\
0.8 & 23.5 & 99.3 \\
0.9 & 0.0 & 100.0 \\
\hline
\end{tabular}

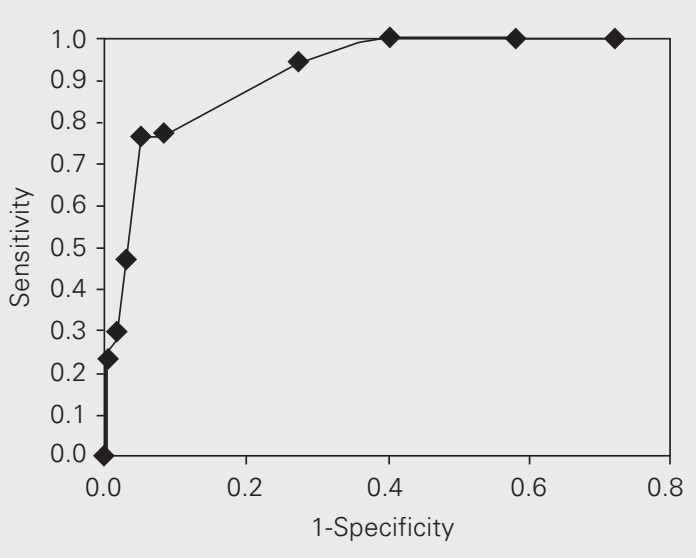

Figure 2. The receiver operating characteristic curve. 
for the system output score was reinforced by the results observed in the study sample, which conferred the fuzzy expert system a sensitivity of $76.5 \%$ and a specificity of $94.8 \%$ for the prediction of the need for ANRM. The plotting of the ROC curve (scores 0 to 10) is shown in Figure 2. The area under the ROC curve was 0.93 (95\%CI: 0.90-0.96).

\section{Discussion}

Several good clinical articles have been published in the last decade regarding the risks and consequences of perinatal asphyxia and the benefits of implementing standards of neonatal resuscitation procedures in hospitals $(24,25)$.

As appropriately observed by Stool and Measham (25), according to the World Health Organization, approximately $32 \%$ of all neonatal deaths in developing countries - equivalent to about 4,500 daily deaths - are due to birth asphyxia and birth injury. And these events are not only frequent causes of mortality and long-term morbidity, but are potentially preventable.

The basis of the present study is the early identification of risky clinical conditions as a way to anticipate the necessity of skilled personnel for patient care. Quoting the American Academy of Pediatrics from the international consensus on neonatal resuscitation, we could say: "Anticipation, adequate preparation, accurate evaluation, and prompt initiation of support are the critical steps to successful neonatal resuscitation" and "Appropriate preparation for an anticipated highrisk delivery requires communication between the person(s) caring for the mother and those responsible for resuscitation of the newly born" (24).

It should be pointed out that risk factors dependent on the use of sophisticated and usually expensive antenatal or intrapartum semiologic techniques to assess fetal wellbeing (fetal blood flow by Doppler ultra- sound, cardiotocography, fetal scalp blood $\mathrm{pH}$ measurement, etc.) were intentionally excluded from our system development as a way to make it useful to any health care team in hospitals providing care of any complexity level. The expected contribution of the present paper is the proposal of the use of an expert system to help decision making in perinatal care in places where highly skilled specialists and sophisticated equipment are not available. The system can be used even with a cheap outdated 486-processor personal computer or with a portable hand-held notebook. In contrast to Bayesian systems, the fuzzy expert system described here can deal with any number of variables without loss of performance.

In addition to being used for care planning, the fuzzy expert model presented here could be used as a teaching or training tool, helping midwives, residents and medical students to identify and evaluate clinical risk factors.

To the best of our knowledge, this is the first study that proposes the use of the fuzzy sets theory to predict neonatal resuscitation. After an extensive search in the indexed literature we were unable to find similar previously published studies, a fact that prevents comparison of the present findings to those obtained with other approaches. We also did not find any study of prediction of neonatal resuscitation using weighted risk factors.

In the analysis of the efficacy of our system in identifying the need for resuscitation in the cases studied, we considered the less than ideal sensitivity of $76.5 \%$ to be a possible reflex of the known unpredictability of some cases of perinatal asphyxia and a good challenge for future improvement of our model. Since this model was based on information related to risk factors alone, one of the possible ways of improving it would probably be the analysis of the impact of associations of these risk factors.

Despite the promising results obtained with the fuzzy model proposed, other aspects of our findings need additional consid- 
eration. Eighteen of the factors studied were not tested by experimental analysis, for which testing in a multicenter study or over a very long period of time in a prospective study would be probably needed. However, the overall performance of the system seemed very promising, taking into account that some medical procedures (induction of labor and cesarean sections) probably modified the expected natural evolution of cases at risk, causing a reduction in the need for ANRM. For obvious ethical reasons, this issue can never be cleared up.

It is possible that characteristics of the study population and regional obstetric clinical practice habits may affect the importance attributed to some variables, a fact that could be clarified by similar studies conducted at other centers.

These preliminary results suggest that fuzzy relations should be considered as a good method to deal with the imprecision of diagnostic and decision making procedures in perinatology and encourage us to conduct further studies and to refine this model, which is intended to implement an auxiliary system able to help health care staff to make decisions in the delivery room.

\section{References}

1. World Health Organization (1998). Basic newborn resuscitation: a practical guide. WHO/RHT/MSM 98.1 (online). WWW URL: http:// www.who.int/reproductive-health/publications/MSM_98_1/ IVISIVI_98_1_abstract.en.htmI. Accessed February 2003.

2. Chandra S (1997). Perinatal asphyxia: multivariate analysis of risk factors in hospital births. Indian Pediatrics, 34: 206-212.

3. Kolatat T, Vanprapar N \& Thitadilok W (2000). Perinatal asphyxia: multivariate analysis of risk factors. Journal of the Medical Association of Thailand, 83: 1039-1044.

4. Goodwin LK \& lannacchione MA (2002). Data mining methods for improving birth outcomes prediction. Outcomes Management for Nursing Practice, 6: 80-85.

5. Mahieu LM, De Muynck AO, De Dooy JJ, Laroche SM \& Van Acker KJ (2000). Prediction of nosocomial sepsis in neonates by means of a computer-weighted bedside scoring system (NOSEP score). Critical Care Medicine, 28: 2026-2033.

6. Saliba RM, Annegers FJ, Waller DK, Tyson JE \& Mizrahi EM (2001). Risk factors for neonatal seizures: a population-based study, Harris County, Texas, 1992-1994. American Journal of Epidemiology, 154: 14-20.

7. Shankaran S, Fanaroff AA, Wright LL et al. (2002). Risk factors for early death among extremely low-birth-weight infants. American Journal of Obstetrics and Gynecology, 186: 796-802.

8. DeCaunes F, Alexander GR, Berchel C, Guengant JP \& Papiernik E (1990). Anamnestic pregnancy risk assessment. International Journal of Gynecology and Obstetrics, 33: 21-27.

9. Gueorguieva RV, Sarkar NP, Carter RL, Ariet M, Roth J \& Resnik MB (2003). A risk assessment screening test for very low birth weight. Maternal and Child Health Journal, 7: 127-136.

10. Richardson DK, Gray JE, McCormick MC, Workman K \& Goldmann DA (1993). Score for neonatal acute physiology: a physiologic severity index for neonatal intensive care. Pediatrics, 91: 617-623.

11. Richardson DK, Corcoran JD, Escobar G \& Lee SK (2001). SNAP-II and SNAPPE-II: Simplified newborn illness severity and mortality risk scores. Journal of Pediatrics, 138: 92-100.

12. Mahfouf M, Abbod MF \& Linkens DA (2001). A survey of fuzzy logic monitoring and control utilization in medicine. Artificial Intelligence in Medicine, 21: 27-42.
13. Luger GF \& Stubblefield WA (1997). Artificial Intelligence: Structure and Strategies for Complex Problem Solving. 2nd edn. Addison Wesley Longman, Palo Alto, CA, USA.

14. Miller III MC, Westphal Jr MC \& Reigart II JR (1981). Mathematical Models in Medical Diagnosis. Praeger Publishers, New York.

15. Massad E, Burattini MN \& Ortega NRS (1999). Fuzzy logic and measles vaccination: designing a control strategy. International Journal of Epidemiology, 28: 550-557.

16. Ortega NRS, Sallum PC \& Massad E (2000). Fuzzy dynamical systems in epidemic modelling. Kybernetik, 29: 201-218.

17. Nascimento LFC \& Ortega NRS (2002). Fuzzy linguistic model for evaluating the risk of neonatal death. Revista de Saúde Pública, 36: 686-692.

18. Boegl K, Adlassnig KP, Hayashi $Y$, Rothenfluh TE \& Leitich H (2004). Knowledge acquisition in the fuzzy knowledge representation framework of a medical consultation systems. Artificial Intelligence in Medicine, 30: 1-26.

19. Teodorescu HN, Kandel A \& Jain LC (1999). Fuzzy and Neuro-Fuzzy Systems in Medicine. CRC Press, Boca Raton, FL, USA.

20. Velasevic DM, Saletic DZ \& Saletic SZ (2001). A fuzzy sets theory application in determining the severity of respiratory failure. International Journal of Medical Informatics, 63: 101-107.

21. Zadeh LA (1965). Fuzzy sets. Information and Control, 8: 338-353.

22. Klir GJ \& Folger TA (1988). Fuzzy Sets: Uncertainty and Information. Prentice Hall, Englewood Cliffs, NJ, USA.

23. Pedrycz W \& Gomide F (1998). An Introduction to Fuzzy Sets: Analysis and Design. MIT Press, London, UK.

24. American Academy of Pediatrics (2000). International guidelines for neonatal resuscitation - An excerpt from the Guidelines 2000 for Cardiopulmonary Resuscitation and Emergency Cardiovascular Care: International Consensus on Science. Pediatrics (online), 106. Available from: American Academy of Pediatrics. WWW URL: http: //www.pediatrics.org/cgi/content/full/106/3/e29. Accessed February 2003.

25. Stool BJ \& Measham AR (2001). Children can't wait: improving the future for the world's poorest infants. Journal of Pediatrics, 139 729-733. 